\title{
PPROC, an Ontology for Transparency in Public Procurement
}

\author{
José Félix MUÑOZ-SORO a, c, 1 , Guillermo ESTEBAN ${ }^{\mathrm{a}}$, Oscar CORCHO ${ }^{\mathrm{b}}$ and \\ Francisco SERÓN ${ }^{c}$ \\ ${ }^{a}$ Aragonese Foundation for Research \& Development (ARAID, Spain \\ ${ }^{\mathrm{b}}$ Ontology Engineering Group. Facultad de Informática, Universidad Politécnica de \\ Madrid, Spain \\ ${ }^{\mathrm{c}}$ Legal-Business Laboratory of the Tech Park Walqa, Universidad de Zaragoza, Spain
}

\begin{abstract}
Public procurement or tendering refers to the process followed by public authorities for the procurement of goods and services. In most developed countries, the law imposes public authorities to provide online information to ensure competitive tendering as far as possible, for which the adequate announcement of tenders is an essential requirement. In addition, transparency laws being proposed in such countries are making the monitoring of public contracts by citizens a fundamental right. This paper describes the PPROC ontology, which has been developed to give support to both processes, publication and accountability, by semantically describing public procurement processes and contracts. The PPROC ontology is extensive, since it covers not only the usual data about the tender, its objectives, deadlines, and awardees, but also details of the whole process, from the initial contract publication to its termination. This makes it possible to use the ontology for both open data publication purposes and for the overall management of the public contract procurement process.
\end{abstract}

Keywords. ontology, public procurement, open government data, legal institution

\section{Introduction}

\subsection{The Electronic Publication of Information Regarding Public Procurement}

In the context of public procurement, advertising has been always an essential part of the process, as it fulfils a dual purpose: on the one hand, it is a resource to improve competitive tendering and, on the other, it constitutes an instrument for transparency and for the monitoring of the behaviour of the contracting authorities [1]. This second purpose is becoming increasingly important because one of the third-generation human rights is free access to public sector information, which is now included in the laws of the majority of developed countries [2].

With the progress of electronic government, the publication of information regarding contracting procedures increasingly began to be performed using electronic means. For instance, some European directives from 2004 (Directives 2004/17/EC and

\footnotetext{
${ }^{1}$ José Félix Muñoz, Edificio I+D, Walqa Technological Park, E-22197, Huesca, Spain. jfm@unizar.es
} 
2004/18/EC) $)^{2}$ created a specific mechanism called the buyer profile. All public sector entities must publish on it the notices about the contracts that they put out to tender. The most important of these announcements is the contract notice, in which the characteristics of the contract are explained and businesses are invited to compete. Other advertisements report progress of the contract, such as the award or the formalization notices. In consequence, buyer profiles have become the central information hub for companies and citizens when it comes to public procurement. However, its use has been severely limited by the major functional and technical differences between the different profiles of different public authorities and the lack of interoperability among them, what makes the integrated processing of the information published on them very hard (for instance, aggregating the total income for a specific company across all the public authorities in a group of countries). One of the currently adopted solutions for this heterogeneity problem consists in forcing all public authorities to publish on a single website the announcements regarding tender procedures. In Spain this site is the Public Sector Contracting Platform (PCSP, https://contrataciondelestado.es).

This solution may be sufficient in order to comply with the needs of competitive tendering, because it is enough to publish a limited set of announcements. However, transparency requires giving citizens much more information and, in addition, transparency practices can vary greatly depending on the policies followed by each authority. Therefore, from the perspective of transparency, the solution involves preparing standards that may be used by administrations to publish all the information that they consider appropriate. To contribute to the development of these standards, we have created the PPROC ontology (http://contsem.unizar.es/def/sector-publico/pproc) with the aim to publish, in a structured and standardized manner, public procurement information on the buyer profiles of public authorities. This ontology has the potential to improve efficiency (for example, since it would enable computerized consultations of the profiles of the various administrations) and to facilitate access for all parties interested in information regarding public contracts. This means that not only contracting powers and tenderer companies, but also the general public as a whole have been considered in the design of this ontology.

\subsection{Knowledge Representation in Public eProcurement Systems}

One of the tasks prior to the publication of the contracts of the Zaragoza City Council and the Provincial Government of Huesca was the analysis of the information used in their contract management applications. In this analysis, we observed that the structure of the databases of these management systems was closely related to the temporal succession in which the information was being generated or received by the management bodies. This leads to an organization of data that we may call "processoriented", which often appears in the relational databases used by public administrations.

On the other hand, there are various initiatives whose purpose is to create standards for electronic procurement, including, within the scope of the EU,

\footnotetext{
${ }^{2}$ Today replaced by the directives of the European Parliament and of the Council: $2014 / 24 / E U$, of 26 February 2014 on public procurement and repealing Directive 2004/18/EC; and 2014/25/EU, of 26 February 2014 , on procurement by entities operating in the water, energy, transport and postal services sectors and repealing Directive 2004/17/EC.
} 
OpenPEPPOL ${ }^{3}$ and $\mathrm{CEN} \mathrm{BII}^{4}$. In both cases, $\mathrm{XML}$ formats are defined to make it possible to structure the messages exchanged by the various agents involved in electronic procurement. Besides, as we have already mentioned, both the EU and national governments have created web sites whose function is to centrally publish information about public tenders. The announcements that are published on these web sites are among the first exchanges of information performed electronically using structured messages. However, to do this the administrations chose to create "de facto" standards, such as the one established for the TED eSenders or CODICE (https://contrataciondelestado.es/wps/portal/codice), defined for the Spanish PSCP. The objective of these standards is to achieve interoperability, addressing communication between systems. Therefore, its domain is limited to the information that, at any time, is transmitted between the various organizations that are involved in the process. Moreover, the structure of the information is provided by the content of the documents that are exchanged. Consequently, we can call these XML standards "documentoriented".

Unlike the above situations, the development of ontologies should not be based on elements associated exclusively with the procedure - such as the sequence in which information or communications actions are created - or with the message exchange, but with a general, all-encompassing view of the reality to be represented. In our case, we have not aimed at the creation of models of general legal concepts, as it happens in some core legal ontologies [3], but at the modelling of a specific social mechanism, used to connect the contracting process of public sector entities. From the perspective of philosophy of law, this objective is related to the concept of a "legal institution" through which the physical, social and legal elements that comprise a given "social mechanism" are identified and described - such as marriage or contracts, for example - focused on the attainment of a defined objective. According to an approach based on the "theory of the institution" the central focus of the model would be public procurement, considered as a legal institution whose purpose is the attainment of a "product": a public contract [4]. We have considered this approach appropriate because this concept of a legal institution (or of the domain to be represented, which amounts to the same thing) as a group of resources focused on the attainment of an objective, is closely associated with the functional aspects of organizations, as is the case with computer applications or tools. For all these reasons, we can state that the PPROC ontology is "institution-oriented".

This perspective has determined the semantic relationships of the model. Some systems of legal concepts are organised vertically from the most general concepts to the most specific ones. In this case, the relationships are about belonging. Other systems, known as operational families, gather together the elements related to a specific item [5]. An institution-based model belongs to this second type and their semantic relationships are organised according to the role that each concept plays within the "institution" that is represented. In order to identify and define these relationships, the science of the law can be used, which is devoted to studying and organizing the legal elements that comprise institutions and the relationships between them. Therefore, the

\footnotetext{
3 OpenPEPPOL is an offshoot association of the Pan-European Public Procurement Online (PEPPOL) project (http://www.peppol.ue).

4 CEN Workshop on Business Interoperability Interfaces for public procurement in Europe (http://www.cenbii.eu/) is an initiative of the European Committee for Standardization (https://www.cen.eu), an association that brings together the National Standardization Bodies of 33 European countries.
} 
institution-oriented perspective is also appropriate to develop a model whose structure will be in accordance with that defined by legal doctrine. For instance, by following this organization in PPROC, it is possible to differentiate, on the one hand, the objective elements-including the purpose of the contract-from the subjective elements - the parties - ; and, on the other hand, the material elements - which are the elements related to the merits of the matter - from the formal elements - which are the ones related to the proceeding. In accordance with these classification criteria, the ontology is divided into four blocks.

The paper is structured as follows: we start with an analysis of existing ontologies in this domain, namely PCO, LOTED2 and MOLDEAS (\$2), and a description of the ontology development method used $(\S 3)$. The structure and main components of PPROC, as well as its relationships with other ontologies, are described in section $\S 4$. In section $\$ 5$ there is a description of the usage, including the experience of two Spanish public administrations that have been early adopters of this ontology and are now using it in their production environments to publish structured information about public contracts in their buyer profiles. As a conclusion, we describe future works and make some observations about the influence of the adoption of the ontology in the information system of the entities.

\section{Related Work}

In [6] we can see an exhaustive study regarding the numerous initiatives implemented for the use of semantic technologies in e-Procurement. Among these, some have focused on the announcement of information regarding public contracts. In the European context, the first experience was LOTED2 [7], which expanded the LOTED ontology [8] with the goals of (a) expressing the main legal concepts of the public contract announcements defined in legal sources, (b) supporting rich semantic annotation, indexing, search and retrieval of tender documents, (c) making it possible to reuse semi-structured data extracted from the TED system ${ }^{5}$ and (d) enabling the integration with other ontologies and vocabularies about related domains. The ontology bases most of its content on the two directives (2004/17/EC and 2004/18/EC), which at the time of development regulated public contracts in Europe, and is the result of a thorough study of legal documents. This means that the legal content of European procurement is heavily present and rigorously represented in LOTED2.

Another initiative focused on public procurement is MOLDEAS (Methods On Linked Data for E-procurement Applying Semantics) [9], an ontology focused on the representation of information contained in the announcements about public tenders. The objective of this ontology was to provide a pan-European standard about public procurement data, enriching it with the classifications of already-existing products and publishing it by following established open data guidelines.

A third initiative in the EU is the Public Contracts Ontology (PCO), implemented within the framework of the LOD2 Project [10]. One of its objectives was to demonstrate the application of Linked Data for the publication of Linked Data about contracts in the public sector, by emulating the market process of meeting supply and demand in order to produce a "business impact". With this purpose in mind, PCO

\footnotetext{
5 The Tenders Electronic Daily (http://ted. europa.eu) is the online version of the Supplement to the Official Journal of the EU, dedicated to public procurement.
} 
models the main aspects of public contracts, although not in great depth. The ontology considers "only the information that is publicly available in existing systems on the Web [...], mainly produced during the tendering phase". Hence, the result is a lightweight ontology that reuses widely accepted ontologies and vocabularies such as VCard, Payments Ontology, schema.org, Call for Anything vocabulary and GoodRelations.

These three ontologies differ in two main aspects. One is the source of the ontology knowledge. PCO and MOLDEAS use diverse sources of information, trying to identify an information core of the domain of public contracts, describing the main concepts of public procurement without delving much into details. In the case of LOTED2, focused on defining a complete legal ontology, its main sources of information are European directives. The second aspect - the complexity of the ontology - is closely related to the first one. PCO defines most of the information that a public contract may need, but some specific relations, roles or behaviours are not strictly represented (e.g. the contracting body or distinguish between objective and subjective award criterions). On the other hand, LOTED2 represents almost every aspect of public procurement, including the properties needed to label information from the TED web site, with the result that his the model is closely related to the text of the 2004 directives.

After studying these ontologies, the decision to develop the PPROC ontology was taken. On the one hand, because two of them (PCO and MOLDEAS) had not the degree of detail required for project purposes. On the other hand, LOTED2 model was considered too complex. In addition, we consider that their model was excessively centered in legal texts. Nevertheless, the main reason for undertaking a new development was that the main objective we chased with the use of the ontology was to improve the transparency of public contracting processes. Under this perspective, none of the three ontologies studied was satisfactory, because they had not transparency as its primary goal. In consequence, they did not detail many of the public procurement concepts that are necessary for transparency purpose and they were not designed to facilitate the understanding by citizenship of the information provided in the buyers profile.

\section{Ontology Development}

\subsection{Requirements}

Our ontology has been developed following the method proposed by Noy [11]. According to this method, the first step is to define the scope of the ontology. In a first approximation, the knowledge required to express the information published on the buyer profile was identified. We defined a clear scope of the size and complexity suitable to deal with the development of the ontology. It is important to bear in mind that the ontology has a twofold purpose: provide the information recorded in the buyer profile of public organisations, and as a tool focused on transparency, all of the relevant information regarding the procurement procedures.

We also defined the territorial scope, which was the European Union. However, as this is a fairly detailed model, there are terms (approximately 20\%) that refer to particular features of Spanish law and even regional particular features within this. This fact does not represent an obstacle for the use of the ontology in other EU countries, as 
we basically describe the model established through directives, and users can ignore or adapt the particular features when there are none or when they are applied differently in their country. Similarly, the ontology can be used in Latin American countries, as there is a fairly close affinity between Spanish legislation and their legislation, which also includes an announcement mechanism that is equivalent to the buyer profile [12]. Regarding the possible use of PPROC in other countries, the international regulatory text with the greatest scope is the Agreement on Government Procurement, of the World Trade Organization (www.wto.org/english/docs_e/legal_e/gpr-94_02_e.htm), which does not establish a detailed regulation of procurement procedures.

In the specification of final users, we considered the following: contract authorities, suppliers, citizens, control authorities, and researchers and agencies interested in the study and analysis of public procurement. Below, we identify several of the competency questions that the ontology should solve, provided by several stakeholders who wanted to make use of the data that was going to be published for different purposes.

- First 50 contracts with most budget

- List of the latest contracts awarded

- List contracts by type

- List contracts by procedure

- List of contracts grouped by managing department (i.e. water and sewer, gardening)

- List of suppliers that have worked with public authorities in the year 2014

- List of steps taken by a contract

- Number of formalized contracts between 11/11/2011 and the current date

- Actual price of all the contracts started, awarded or formalized in 2011, 2012 and 2013

- Total price of the formalized contracts with the supplier MULTITEC

- Id, subtype and date of the formalized contracts with the supplier URBANCO

- URI and names of the managing department with the largest amount of contracts

Finally, we identified the ontological and non-ontological resources to be used. The non-ontological resources included the Common Procurement Vocabulary (CPV, $\mathrm{http} / /$ simap.europa.eu/codes-and-nomenclatures/codes-cpv/codes-cpv_en.htm). It is exclusively used in EU procurement and consists of a main vocabulary for defining the object of a contract without entering into great detail, and a supplementary vocabulary for adding further qualitative information. There are also some implementations of this vocabulary in RDF, like the one available at http://cpv.data.ac.uk or the one described by Alvarez-Rodríguez and colleagues [13]. On the other hand, the reused ontologies are: Public Contracts Ontology (PCO), Organization Ontology, Friend Of A Friend (FOAF, http://www.foaf-project.org/), SKOS [13], schema.org, Dublin Core Metadata Terms (dcterms) and Good Relations [14].

We have made available the Ontology Requirement Specification Document (ORSD), that we initially built following our ontology engineering methodology, in figshare (http://dx.doi.org/10.6084/m9.figshare.1314591). For completeness and better understandability purposes, this ORSD is also linked from the ontology (both in the HTML documentation and as a value of the rdfs: seeAlso property associated to the ontology). 


\subsection{Development}

The enumeration of the relevant terms for the model was performed with two sets of stakeholders. On the one hand, the company iASoft, which has developed the buyer profiles of numerous Spanish administrations, compiled the fields included in the various documents published on buyer profiles. On the other hand, several legal experts analysed the annexes of the European directives and Spanish legislation that specify the announcement models for their publication. Then, the ontology development team consolidated this information and used it as a basis to prepare an initial list of entities, including cardinality, domain and range for properties. This list had 111 first-level entities, and the team divided some of them into several second-level entities, of which there were 42 in total. This approach was completed and validated by the contracting managers of three public administrations, the Zaragoza City Council, the Provincial Government of Huesca and the Regional Government of Aragón.

At the same time, the classes and properties present in other ontologies that may be used to describe entities at a higher level were identified. Later, in order to link this set of entities to each other, in a joint task between ontology developers and legal experts, classes and properties were defined to organize the contents according to their nature and function. Therefore, although the strategy was basically bottom-up, as it started from the most detailed elements present in the buyer profiles and in the annexes to prepare an initial approximation of the ontology, an effort was also made to make them consistent with the highest-level concepts defined in other ontologies. Finally, the ontology was implemented in OWL.

Another question to be determined during ontology development was which classes should be declared of mandatory use and which of recommended use. The sources of knowledge used did not resolve the question, as laws do not establish that there are fields that must necessarily be published and the practice of the different administrations varies considerably. In the absence of these sources, the CODICE standard was taken as a reference for Spain. Here, the properties that relate to the following ontology classes are declared as mandatory: dcterms:title, pc:contractingAuthority, pproc:awardDate, pc:tender, pc: supplier y dcterms:identifier.

These tasks were carried out during 2013 and the beginning of 2014. In April 2014, two of the aforementioned public administrations (Zaragoza City Council and the Provincial Government of Huesca) started labelling their buyer profiles according to the PPROC ontology, producing instances of the different classes and properties of the ontology. This activity served as a basis for a review of the ontology, which was carried out jointly by the legal experts, the public administrations and the ontology development team. The objective of the review was to fulfil the labelling expectations of the public administrations whilst maintaining the legibility of the model. Finally, at the end of 2014 the PPROC 1.0.0 version was published. 


\section{Ontology Description}

\subsection{The pproc:Contract Class}

The class pproc: Contract is the main class for the definition of a contract. It contains the basic information about the contract and serves as an entry point to link to the other classes. We define pproc:Contract as a subclass of pc:Contract. We have taken PCO as a starting point for the preparation of PPROC and the pc:Contract class is used as the domain of data properties such as dcterms:title, pc:tenderDeadline or pc:actualEndDate among others. Many of these properties are being reused in the PPROC ontology and thus do not require changes. However, many other properties are rewritten, as PPROC has specific classes to describe these properties. The reason is that we provide a separate class for each kind of element, which helps ontology users to better define the scope of searches. Some PPROC classes are aligned towards PCO, and we define them as rdfs: subclassof of their corresponding PCO classes.

Public contracts may belong to many different categories (they can be contracts with and without lots, extendable, multiannual, etc.). Initially, we considered two different alternatives to categorize these types of public contracts: SKOS classification schemes (e.g. the PCO ontology uses two of them for this purpose: pc: ProcedureTypeScheme and pc:KindScheme, to which pc:Contract is linked through the properties pc:procedureType and pc:kind respectively), or several class taxonomies for the different types of contracts. We have opted for the latter option (creating several class taxonomies to replace the use of pc: KindScheme), because (a), we wanted to specify more clearly the different types of contracts that we may have to deal with and (b), we added some class restrictions relevant for some of these classes (e.g. pproc:ContractwithLots owl: subclassof pproc:lot some pproc:Lot).

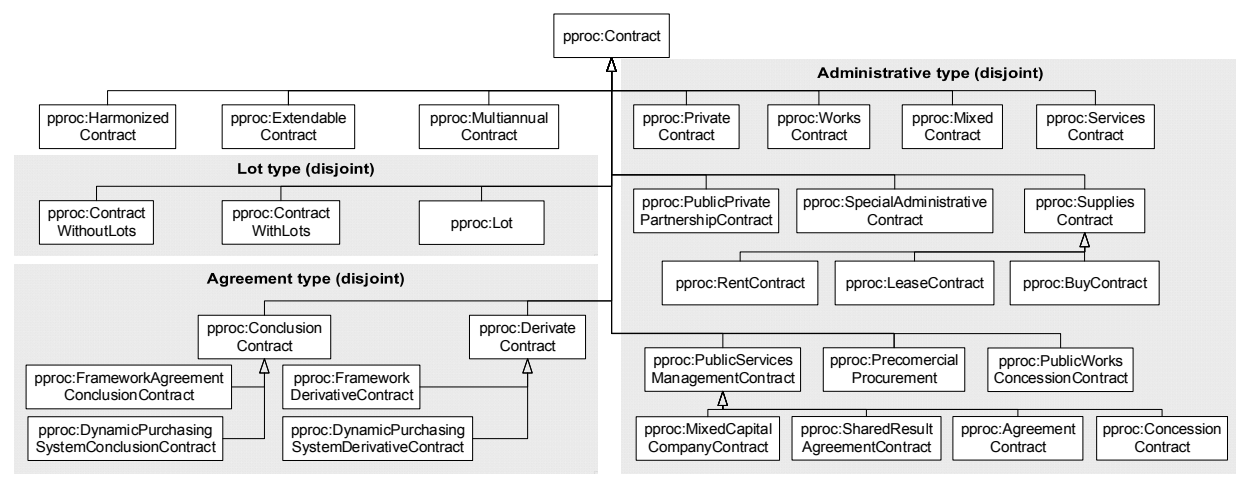

Figure 1. Classifications of the pproc: Contract class

Furthermore, contracts are not only classified according to their administrative type. Contracts can be also extendable, harmonized (i.e. reaches the threshold of regulation of European procurement directives), private or multiannual, etc. (see Fig. 1). Besides, contracts may be subdivided into lots. Each lot is a contract in itself, with a defined object and that can be awarded separately, but that forms part of a main 
contract. PCO instantiates contracts, with or without lots, and lots as pc: Contract; that is, this class is the domain and range of the property used to relate contracts to their lots (pc: lot). In PPROC (as in LOTED2) specific classes for each of the cases are created.

As an example of the use of the ontology, we include the representation in JSONLD of a supply contract for the acquisition of training equipment for the fire brigade of the Zaragoza City Council. The representation begins with some properties linked directly to the pproc: Contract class.

\{

"@context" : "http://contsem.unizar.es/docs/context.jsonld",

"@id" : "contzar:0308848-14",

"@type" : [

"pproc:Contract"

"pproc:SuppliesContract",

"pproc:HarmonizedContract",

"pproc:MultiannualContract",

]

"pproc:ContractWithoutLots"

"terms:title" : "Fire training system",

"dcterms:identifier" : "0308848/14"

\}

The PPROC ontology is also composed of other 77 classes and 129 properties that make it possible to represent the contract and the procedure for its preparation. For the representation below, these classes are divided into four blocks, each one of which includes the classes that are directly related to the following points of the contract: 1) the object, which is the supply that the contract covers; 2) the parts, which are the agents that participate in the procurement process and, when appropriate, in the contract (the contracting authority, tender, awarded tender, etc.); 3) the procedure, composed of the steps taken until the execution of the contract; and 4) the fulfilment, which includes actions that must be taken after the execution. Below we show the main classes that comprise each block, some of the decisions taken in the design and, when appropriate, the relationship with other ontologies.

\subsection{Contract Object}

Two different (non-exclusive) approaches can be used to define the product or service wich forms the contract object. The first one consists in using the pproc:object or pc:mainObject properties, which are especially appropriate for using the Common Procurement Vocabulary (CPV). This option must necessarily be included in the ontology, as the use of the CPV is mandatory in contracts subject to harmonised regulation and the control bodies recommend its use in all contracts. However, the scope of the CPV is restricted to public procurement, meaning that it is advisable to have a second way of describing the products and services for extended use in eProcurement. With this objective in mind, the gr : Offering class of GoodRelations is used. In addition, it must be remembered that, although in the majority of contracts the contracting authority receives the service and pays a price for it, there are also contracts in which it is the authority that offers a service (the provision of a public service, for example) and receives a financial consideration. 


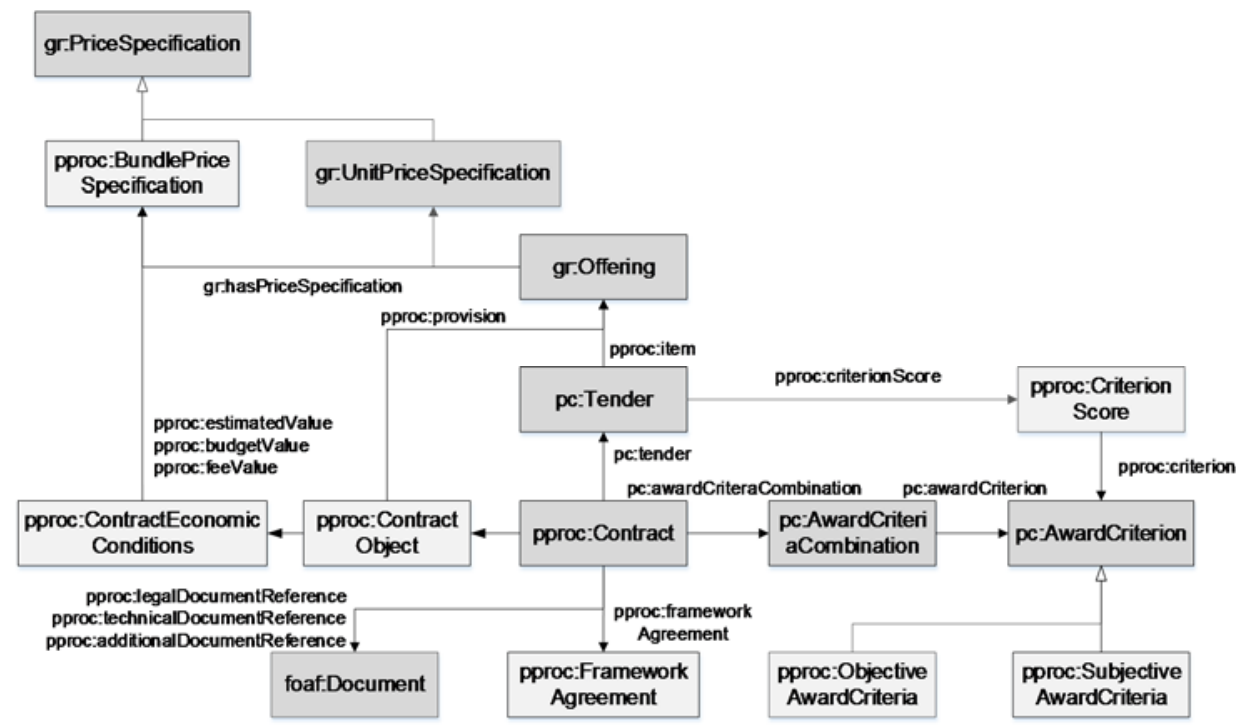

Figure 2. Contract object related clases

To describe prices and payment options we use the gr:Pricespecification class. However, when defining the price of a contract, a bundle of objects or a tender we find that $g r$ :Pricespecification and its known subclasses are not enough to describe some prices. Usually a gr : Offering contains a set of items the contracting authority is going to acquire. Its price can be set using the gr: UnitPriceSpecification class that defines a price of a single item of the offering (e.g. an offering contains 100 printers with the gr:UnitPricespecification specifying the price of a single printer). However, usually it also needs to define the prices of a set of objects as a whole, treating them as a single package, and to this end, PPROC includes the pproc: BundlePricespecification class.

The service covered by the contract can be modified by conditions that are included in this block. The classes that describe the criteria that will be used to award the tender also form part of the block. These are divided into objective criteria (such as the price or the delivery period), which can be quantified and applied through automated systems or by holding auctions, and subjective criteria, which are valued by experts.

\{

"pproc:Contractobject" : \{

"@id" : "contzar:0308848-14/Contractobject",

"@type" : "pproc:Contractobject",

"pproc:mainObject" : "cpv:code-44212320",

"pproc:provision" : \{

"@id" : "contzar:0308848-14/0bject",

"etype" : "gr:offering",

"dcterms:title" : "Fire training system using industrial platform to Zaragoza

\},

City Fire Brigade"

Linked data CPV code of the

object of contract and the name of the contract object 


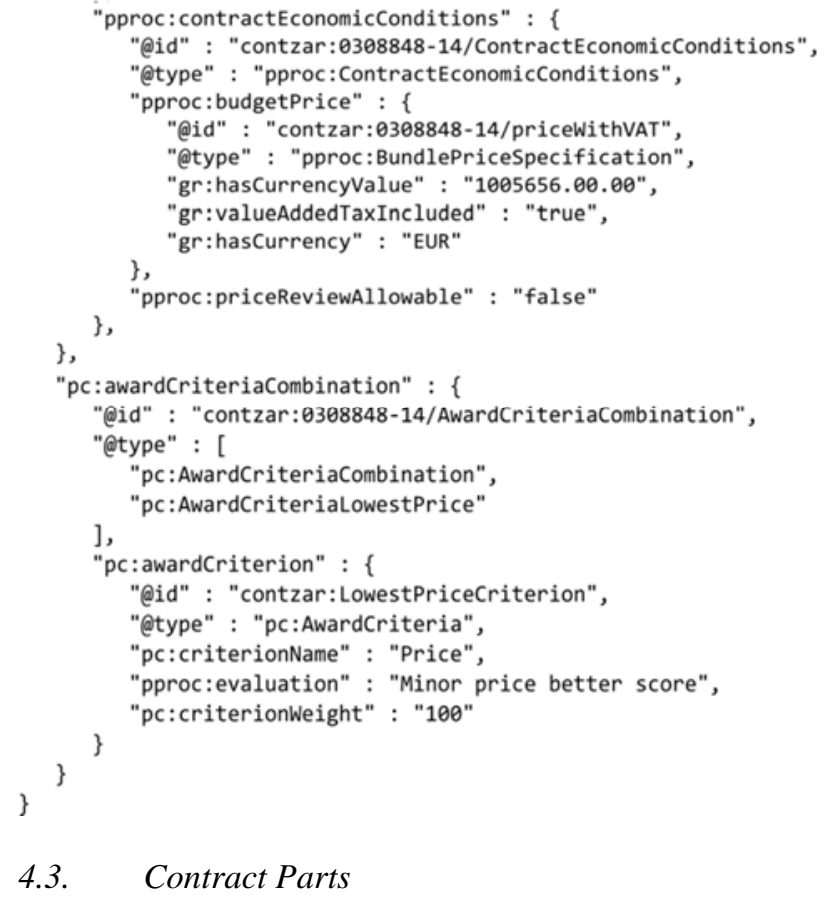

\section{3. $\quad$ Contract Parts}

To describe the parts involved in a public procurement procedure we use the Organization Ontology, which includes the classes and properties needed to describe organizational structures and their hierarchy, through the org:Organization class and org: suborganizationof property. The role that an organization plays in a given procedure or contract-contracting authority, delegating authority, the organization on whose behalf of a contract is being made, the contracting body, managing department and the specific supplier of a tender (tenderer) - is established by the property used to link it with the contract (see Fig.3). Note that in most cases we were able to use the org:suborganizationof property to describe the relation between the contracting body and the managing department belonging to a contracting authority.

To describe the persons grouped together to perform a task of the procedure, we use the pproc:Committee class. These committees are known as contract bodies and may have different functions in the procedure. To differentiate them according to these functions, PPROC includes three subclasses of pproc: Committee. Members belonging to a concrete organization or committee are stated by using membership properties such as $s:$ member or org:memberof. Here we propose using properties belonging to other ontologies and vocabularies, such as the Organization Ontology itself, schema.org, Friend Of A Friend or SKOS. There are several contents where the location or a specific place should be known (e.g. the office of the contracting authority or a tenderer, the location where the goods should be left or the place of a meeting), and to define them we propose using the S:Place class and properties of schema.org. 


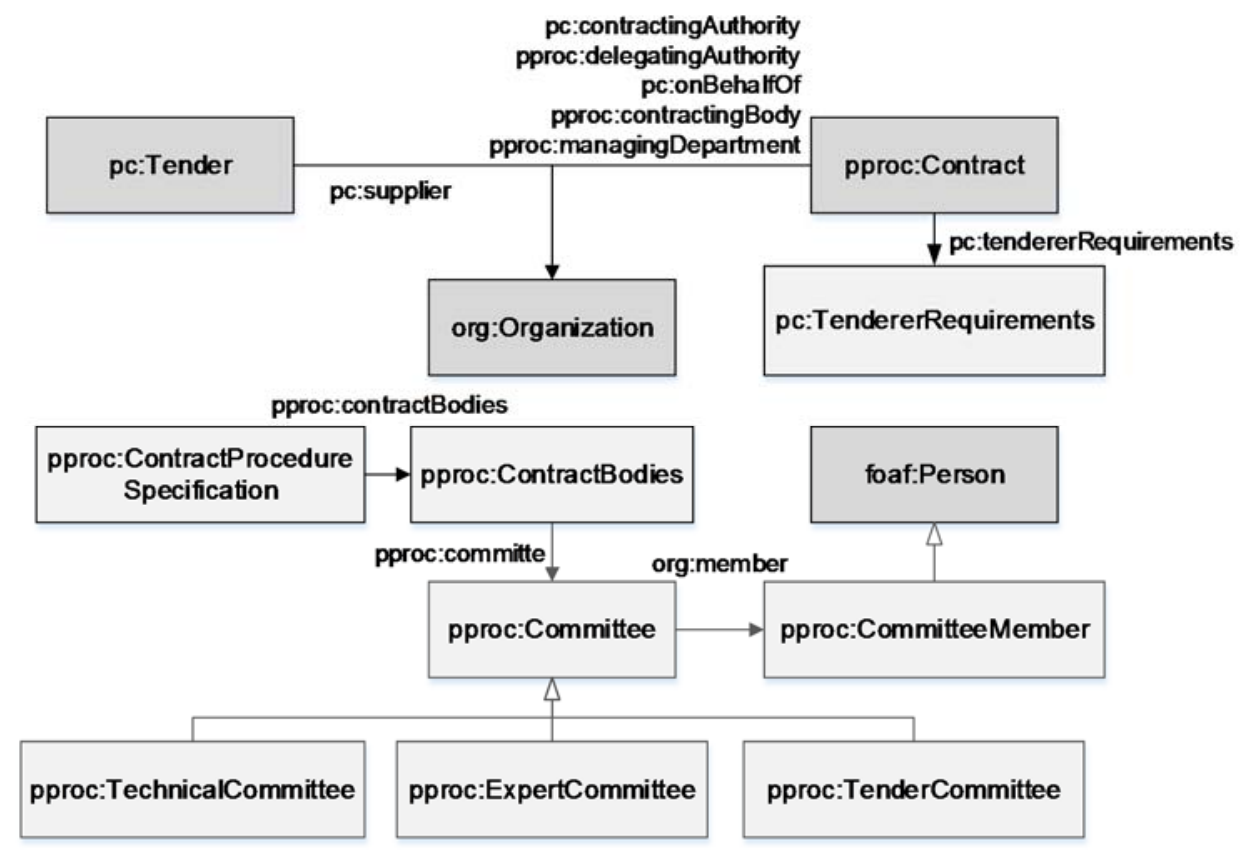

Figure 3. Parts-related classes

Next, the class pc:Tender is reused to describe proposals made by suppliers. PCO uses two properties to link tenders to their related contract: pc:tender and pc: awardedTender. Once again, we believe that the properties defining the nature of an object can be replaced by defining the rdf:type of such an object. Therefore, although we still use the pc: tender property, we created subclasses to further define a pc:Tender (pproc:AwardedTender, pproc:AcceptedTender, pproc: ExcludedTender and pproc: FormalizedTender). Finally, tenderers are also defined through the Organization Ontology and linked using the pc: supplier property from a pc: Tender.

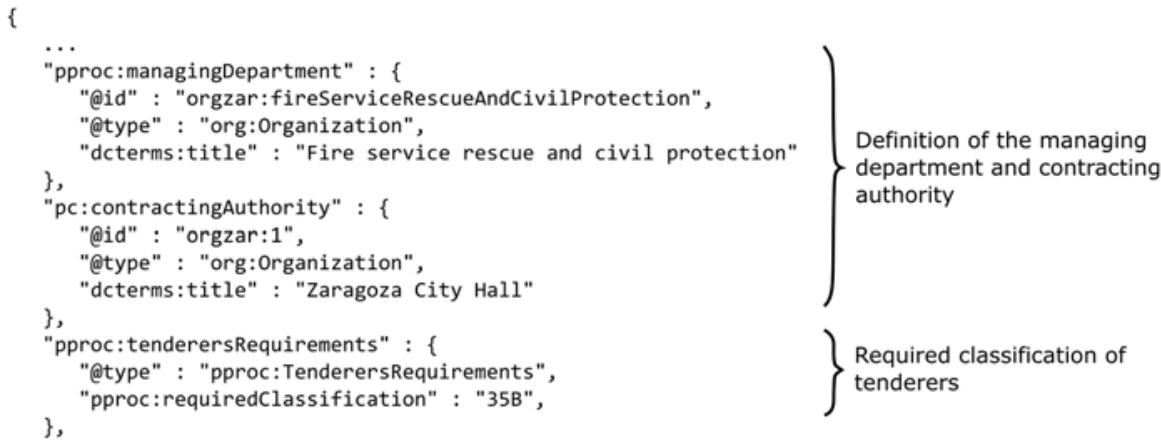

Definition of the managing

department and contracting

authority

Required classification of tenderers 


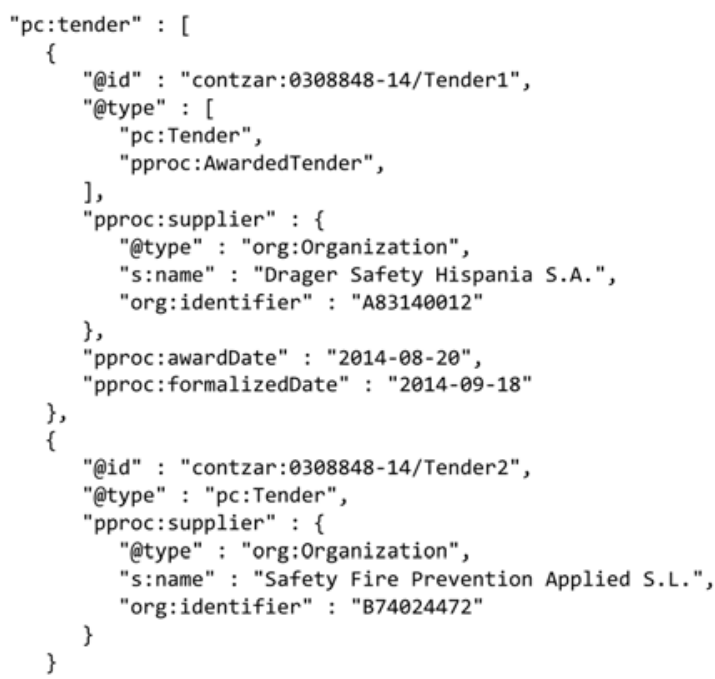

\subsection{Procedure and Fulfillment}

Another block of information is referred to the procedure followed during the procurement process. A first aspect to represent is the kind of procedure and, in contrast to the decision made while classifying contracts and tenders, we use SKOS to define the procedure kind and its urgency, using two concept schemes (pproc: ProcedureTypeScheme and pproc:UrgencyTypeScheme). The use of SKOS is justified in this case because of its simplicity for this type of information where we do not have specific properties and property restrictions applicable to the different types of contracts and tenders. Both properties determining the procedure and urgency have pproc: ContractProcedurespecification as their domain.

We included in PPROC all the information about it that could be useful to suppliers, such as tender requirements or briefing meetings. However, the information about the procedure is very important for the control of contracting and, therefore, the ontology also includes classes to describe other points, such as the people that participate in the procedure or possible resources and their result (see Fig. 4). It is also necessary to know if the type of procedure used is the one related to the contract, and the ontology has specific properties to do this, such as pproc:Assumption protecting the chosen procedure type.

Also, the term of the contract does not end with the execution, which is the time when the contracting procedure is considered as finished. Contracts are often modified at a later time through specific procedures, which often change points such as the price or the term for completion. These modifications can be used to breach the principles of the contracting and, therefore, a fourth block is dedicated to this phase, which we call fulfilment. This contains classes that make it possible to represent the conditions and limits that possible modifications to the contract are subject to. If these occur, they can also be represented through the pproc: ContractModification class. Finally, it also includes classes to include the final result of the contract. 


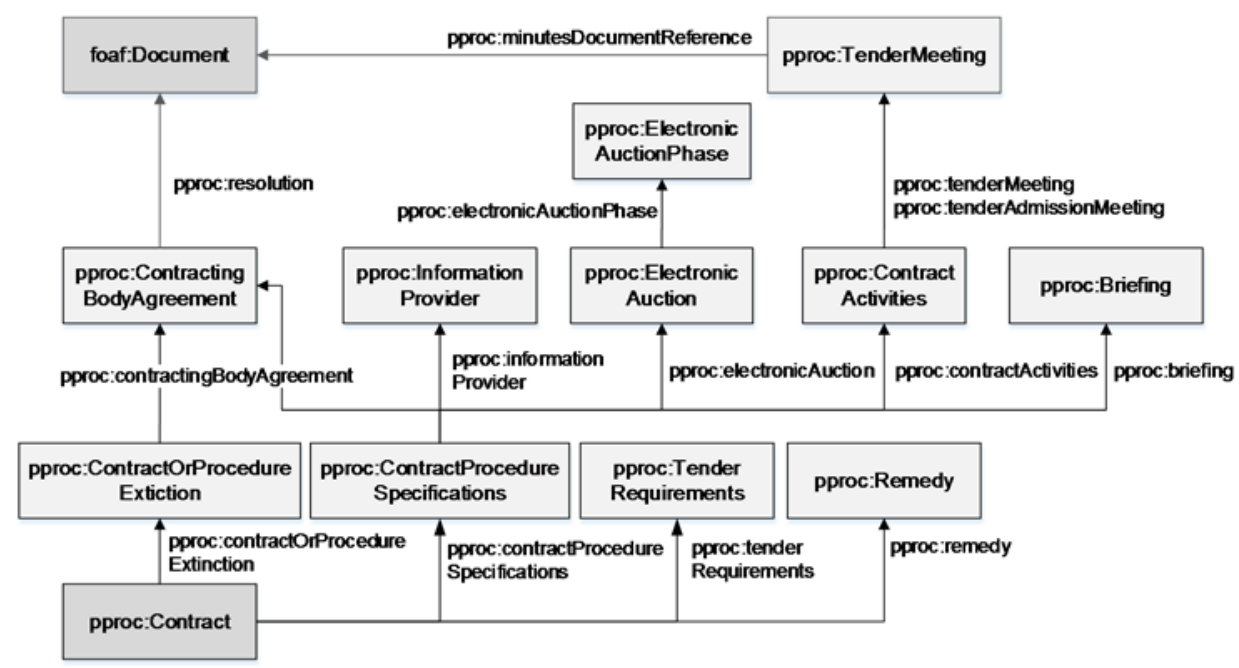

Figure 4. Procedure- related classes

Regulation regarding these classes varies in the different territorial scopes. For example, the legislation for the Aragon region requires the publication of modifications to contracts, which is not the case with the Spanish legislation (although it will probably require it in the next reform of the law), or with European legislation. However, in any case, the block was considered necessary because the publication of modifications should be considered as good practice regarding transparency.

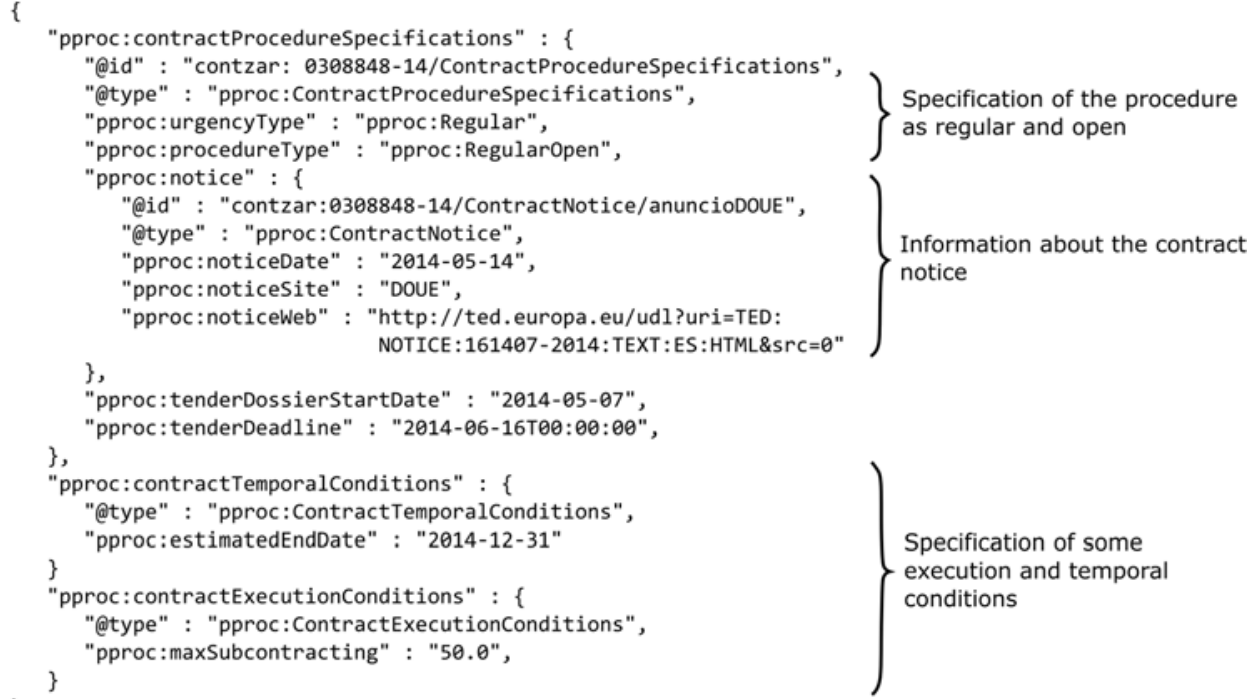




\section{Usage and Evaluation}

PPROC is already being used by two public authorities of different size and scope (Zaragoza's City Council and the Provincial Government of Huesca), which are publishing open data about their public contracts so that they can be used not only by potential tenderers but also by citizens for transparency purposes. In both cases the corresponding PPROC-based RDF data is stored in a SPARQL endpoint (http://www.zaragoza.es/ciudad/risp/sparql.html and http://www.dphuesca.es/sparql respectively), which can be queried for complex information needs. Additionally, in the case of Zaragoza, contracts are offered through their city API, providing data in JSONLD (as described in previous example), as well as in other common formats like JSON, CSV, or XML. Finally, the whole buyer profile of the Zaragoza's City Council (http://zaragoza.es/ciudad/gestionmunicipal/contratos/) is now completely based on the usage of PPROC-based data. At the time of writing, the Regional Government of Aragon has also begun work in order to publish its buyer profile by using the ontology.

Regarding its future use by local administrations, it is important to mention that PPROC is recommended as the ontology to be used by smart cities offering their public contract data according to the proposed technical norm from the Spanish Association for Standardization and Certification (AENOR), UNE 178301 on Open Data for Smart Cities. This technical norm is also proposing the use of other nine vocabularies for the publication of data on transport, tourism, air quality, businesses, etc., and has been jointly developed by a combination of private companies, cities and other regional and national public administrations in Spain.

The ontology is also used to publish information about public sector contracts in Spain as a whole. To do this, a mapping with the CODICE XML standard has been performed, and a continuous transformation process is being carried out. These are published at http://pproc.unizar.es:8890/sparql/, which as of February 2015 contains 12 million RDF triples, including information about 199,611 public sector contracts.

All of the competency questions about the contracting procedures defined in the ORSD have been transformed into SPARQL (https://github.com/pproc/pproc-sparql) and can be resolved using the ontology. For example, the enquiry "Count of Contracts by Type", which in SPARQL is

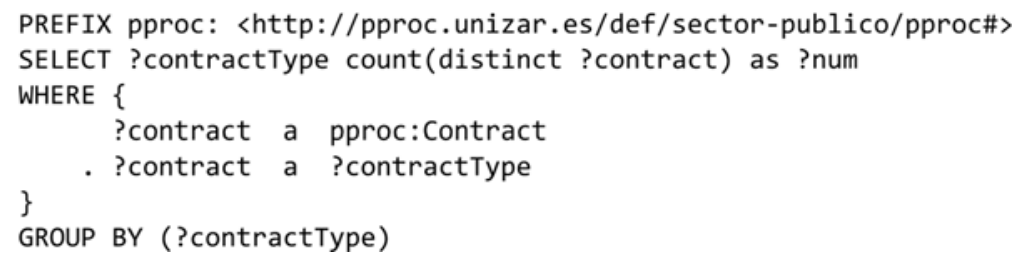

executed with the SPARQL endpoint that contains the public sector contract platforms, provides the following result:

\begin{tabular}{|l|r|}
\hline \multicolumn{1}{|c|}{ contractType } & \multicolumn{1}{c|}{ num } \\
\hline http://contsem.unizar.es/def/sector-publico/pproc\#ServicesContract & 85406 \\
\hline \hline http://contsem.unizar.es/def/sector-publico/pproc\#WorksContract & 19189 \\
\hline \hline http://contsem.unizar.es/def/sector-publico/pproc\#PublicWorksConcessionContract & 135 \\
\hline \hline http://contsem.unizar.es/def/sector-publico/pproc\#RentContract & 15680 \\
\hline
\end{tabular}




\begin{tabular}{|l|r|}
\hline http://contsem.unizar.es/def/sector-publico/pproc\#PrivateContract & 3601 \\
\hline http://contsem.unizar.es/def/sector-publico/pproc\#BuyContract & 46275 \\
\hline http://contsem.unizar.es/def/sector- & 268 \\
\hline publico/pproc\#DynamicPurchasingSystemDerivativeContract & \\
\hline http://contsem.unizar.es/def/sector-publico/pproc\#PublicPrivatePartnershipContract & 25 \\
\hline http://contsem.unizar.es/def/sector-publico/pproc\#SpecialAdministrativeContract & 3856 \\
\hline \hline http://contsem.unizar.es/def/sector-publico/pproc\#FrameworkDerivativeContract & 2300 \\
\hline http://contsem.unizar.es/def/sector-publico/pproc\#Contract & 199611 \\
\hline http://contsem.unizar.es/def/sector- & 27 \\
\hline publico/pproc\#DynamicPurchasingSystemConclusionContract & 47012 \\
\hline http://contsem.unizar.es/def/sector-publico/pproc\#SuppliesContract & 1453 \\
\hline http://contsem.unizar.es/def/sector-publico/pproc\#FrameworkConclusionContract & 1671 \\
\hline \hline http://contsem.unizar.es/def/sector-publico/pproc\#PublicServicesManagementContract & \\
\hline
\end{tabular}

However, as we have already mentioned, the information available in the different administrations varies greatly. Thus, while all these competency questions can be solved in the Zaragoza City Council's SPARQL endpoint, in the case of the Provincial Government of Huesca, only the first four questions could be answered.

The freedom of information acts (FOIA) establishes a set of indicators and data that public entities have to necessarily publish on their websites. Some administrations also have their own rules about transparency, such as the Ordinance of Transparency and Free Access to Information of Zaragoza City Council, which adds a considerable amount of data and indicators to the minimum established by law. We have confirmed as part of our evaluation that the PPROC ontology makes it possible to create SPARQL queries that literally reflect the text of the freedom of information act and the ordinance, such as the following ones:

- Budget volume in percentage of contracts awarded by each of the procedures provided for in the legislation (FOIA).

- A list of all contracts awarded by the City, classified by type and amount, indicating the object, the amount of the bid, the award and the final cost, the procedure for the award, the instruments through which have been published where appropriate, the number of participating tenders, the awarded tenders, the duration or timing of planned and actual implementation, modifications, and any other information of special interest to the public (Ordinance).

This is all covered now at http://www.zaragoza.es/ciudad/gestionmunicipal/contratos/.

\section{Conclusion and Future Work}

In this paper we have described PPROC, an ontology for the description of public procurement that has been developed following standard practices in ontology development, identifying competency questions with different stakeholders (public authorities, companies already working for them and legal experts), and published according to well-established recommendations for Linked Data vocabulary publishing. We have also provided examples of their usage, especially focusing on the generated 
JSON-LD context and a set of SPARQL queries that provide answers to the proposed competency questions.

The ontology is already in use by several public authorities in Spain, and has been recommended as the ontology to be used to publish open data about public contracts in Spain, according to the AENOR technical norm UNE 178301, what will ensure a higher level of uptake in the near future. It has also been included at http://lov.okfn.org/dataset/lov/vocabs/pproc and we are also aligning it currently with the less detailed Open Contracting Standard that is being developed by the World Wide Web Foundation. Future actions to be taken include the adaptation of the ontology to the reform of public procurement that will take place with the transposition of directives 2014/24/UE and 2014/25/UE to the different countries. In Spain there is a draft of the new act at an advanced stage of development, but which has not been made public yet. In any case, we expect that the impact of the modifications on the ontology will not be significant.

Other initiatives focus on the use of the ontology as a resource for the integration of information about contracting procedures. In this regard, work is being carried out in partnership with the Observatory of Public Procurement (http://www.obcp.es/), and a new faceted search engine has been developed that acts on all of the information labelled using PPROC, which already includes over 200,000 contracts. The structure of the data used in the TED system has also been analysed to carry out the mapping using PPROC and the possibility of creating a SPARQL endpoint with this information is being studied.

The appraisal made by the final users of the ontology has been positive. Its adoption has resulted in a major improvement, not only regarding information provided to suppliers and to citizens, but also to the information system of the organisations. Specifically, it has facilitated the integration of information about contracts, which used to be managed in various services and through different applications, and has made it possible to give information a structure that is closely linked to the knowledge and terminology used by experts in procurement. PPROC has been developed based on the law, and that makes interdisciplinary work between engineers and legal experts easier during the development of the ontology and, once it is finished, more understandable for legally-trained users and possibly for everyone else too.

Acknowledgements. PPROC was developed through the project "Optimization of public procurement using semantic technologies (ContSem)", led by the company iASoft (Oesia) and funded by the Spanish Ministry of Industry, Commerce and Tourism (TSI-020606-2012-4). The authors would like to thank Miguel Angel Bernal (co-author of the ontology), and Carlos Bobed and Carlos Becana (contributors) for their cooperation in the development of PPROC. Also to those responsible for procurement and the buyer's profile and the technicians of the participating administrations in the project: the Zaragoza City Council (Maria Jesús Fernandez, Ana Budría, Laura Fernando, Victor Morlán and Rubén Notivol), the Provincial Government of Huesca (Cristina de la Hera, Montserrat Rodríguez and Javier Casado) and the Regional Government of Aragón (Ricardo Cantabrana, José María Subero and Eva Sanz). 


\section{References}

[1] Miroslav, M., Miloš, M., Velimir, Š., Božo, D., Đorđe, L.: Semantic technologies on the mission: Preventing corruption in public procurement. Computers in Industry, 65 (5), 878-890 (2014)

[2] Ackerman, J.M., Sandoval-Ballesteros, I.E.: The global explosion of freedom of information laws. Administrative Law Review, 58 (1), 85-130 (2006)

[3] Agnoloni, T., Fernández-Barrera, M., Sagri, M.T., Tiscorni, D., Venturi, G.: When a FrameNet-Style Knowledge Description Meets an Ontological Characterization of Fundamental Legal Concepts. In: Casanovas, P., Pagallo, U., Sartor, G., Ajani, G. (eds.) AI Approaches to the Complexity of Legal Systems. 93-112. Springer, Berlin (2010)

[4] MacCormick, N.: Norms, Institutions, and institutional Facts, Law and Philosophy, 17, 301-345 (1998)

[5] Fernández-Barrera, M., Sartor, G.: The Legal Theory Perspective: Doctrinal, Conceptual Systems vs. Computational Ontologies. In: Sartor, G., Casanovas, P., Biasiotti M.A., Fernandez-Barrera M. (eds.) Approaches to Legal Ontologies, 15-47. Springer, Dordrecht (2011)

[6] Alvarez-Rodríguez, J.M., Labra, J. E., Ordoñez de Pablos, P.: New trends on e-Procurement applying semantic technologies. Current status and future challenges. Computers in Industry, 65, 800-820 (2014)

[7] Distinto, I., d'Aquin, M., Motta, E.: LOTED2: An Ontology of European Public Procurement Notices. Semantic Web Journal, Reviewed (2012)

[8] Valle, F., d'Aquin, M., Noia, T. D., Motta, E.: LOTED: Exploiting Linked Data in Analysing European Procurement Notices". In: Proceedings of the 1st EKAW Workshop on Knowledge Injection into and Extraction from Linked Data (2010)

[9] Alvarez-Rodríguez, J.M., Labra, J. E., Cifuentes, F., Alor-Hernandez, G., Sánchez, C., Luna, J. A. G.: Towards a pan-European e-procurement platform to aggregate, publish and search public procurement notices powered by linked open data: the MOLDEAS approach. International Journal of Software Engineering and Knowledge Engineering, 22 (03), 365-383 (2012)

[10] Nečaský, M., Klímek, J., Mynarz, J., Knap, T., Svátek, V., Stárka, J.: Linked data support for filing public contracts. Computers in Industry, 65 (5), 862-877 (2014)

[11] Noy, N. F., McGuinness, D. L.: Ontology development 101: A guide to creating your first ontology (2001)

[12] Moreno Molina, J.A., Panorama comparado de la Contratación Pública en América Latina, Observatorio de la Contratación Pública, www.obcp.es, 2015

[13] Alvarez-Rodríguez, J. M., Labra-Gayo J. E., Rodríguez-González, A., Ordoñez de Pablos, P.: Empowering the access to public procurement opportunities by means of linking controlled vocabularies. A case study of Product Scheme Classifications in the European e-Procurement sector, Computers in Human Behavior, 30, 674-688, (2014)

[14] Miles, A., Matthews, B., Wilson, M., Brickley, D.: SKOS core: simple knowledge organisation for the web. In: International Conference on Dublin Core and Metadata Applications, 3-10 (2005)

[15] Hepp, M.: GoodRelations: An ontology for describing products and services offers on the web. In: Knowledge Engineering: Practice and Patterns. Springer, Berlín, 329-346 (2008) 